$40(3) \mid 2011$

Varia

\title{
La mano de obra indígena en el comercio fluvial del Beni (Bolivia) en la segunda mitad del siglo XIX
}

La main-d'oeuvre indigène dans le commerce fluvial du Beni (Bolivie) dans la deuxième moitié du XIX ${ }^{e}$ siècle

The indigenous workforce in the fluvial trade of the Beni (Bolivia) in the second half of the $19^{\text {th }}$ century

\section{Anna Guiteras Mombiola}

\section{OpenEdition}

\section{Journals}

Edición electrónica

URL: http://journals.openedition.org/bifea/1387

DOI: $10.4000 /$ bifea. 1387

ISSN: 2076-5827

\section{Editor}

Institut Français d'Études Andines

\section{Edición impresa}

Fecha de publicación: 1 diciembre 2011

Paginación: 511-532

ISSN: 0303-7495

\section{Referencia electrónica}

Anna Guiteras Mombiola, «La mano de obra indígena en el comercio fluvial del Beni (Bolivia) en la segunda mitad del siglo XIX », Bulletin de l'Institut français d'études andines [En línea], 40 (3) | 2011 Publicado el 01 junio 2012, consultado el 06 noviembre 2020. URL : http://journals.openedition.org/ bifea/1387 ; DOI : https://doi.org/10.4000/bifea.1387

\section{(c) $(1) \odot$}

Les contenus du Bulletin de l'Institut français d'études andines sont mis à disposition selon les termes de la licence Creative Commons Attribution - Pas d'Utilisation Commerciale - Pas de Modification 4.0 International. 


\title{
La mano de obra indígena en el comercio fluvial del Beni (Bolivia) en la segunda mitad del siglo $\mathrm{XIX}^{*}$
}

\author{
Anna Guiteras Mombiola**
}

\begin{abstract}
Resumen
Por decreto del 6 de agosto de 1842 los indígenas benianos adquirieron la categoría de ciudadanos que, además de otorgarles derechos de propiedad, igualdad y libertad, también propició su incorporación como mano de obra a las actividades económicas y comerciales controladas por los grupos blancomestizos que, progresivamente, se desplazaron a la región. La historiografía existente sobre el proceso pone énfasis en la ineficacia del decreto. Por el contrario, el análisis de la legislación estatal y los documentos relativos a la contratación de tripulaciones, nos permite sostener que una parte de la población indígena dedicada al transporte fluvial y las autoridades públicas de la prefectura intentaron conciliar las garantías constitucionales de la población nativa con el desarrollo económico de la región.
\end{abstract}

Palabras clave: Bolivia, Amazonía, indígenas, legislación, derechos, mano de obra

\section{La main-d'oeuvre indigène dans le commerce fluvial du Beni (Bolivie) dans la deuxième moitié du XIX'e siècle}

\section{Résumé}

Le décret du 6 août 1842, a permis aux indigènes du département du Beni d'acquérir la catégorie de citoyens et leur a garanti le droit de propriété, d'égalité et de liberté. Ce décret a permis également aux

* Este trabajo fue presentado en el VI Congreso de la Asociación de Estudios Bolivianos organizado por el Archivo y Biblioteca Nacionales de Bolivia y la Asociación de Estudios Bolivianos, celebrado en Sucre (Bolivia) del 27 al 30 de junio de 2011 y forma parte de la tesis doctoral en curso bajo la dirección de la Dra. Pilar García Jordán y se inscribe en el proyecto de investigación I+D+i del Ministerio de Ciencia e Innovación ref. HAR2009-07094. Agradezco los comentarios realizados por Isabelle Combès y Zulema Lehm a versiones anteriores de este trabajo.

** Universitat de Barcelona, Taller de Estudios e Investigaciones Andino-Amazónicos (TEIAA). E-mail: anna.guiteras@gmail.com 
indigènes de s'offrir comme main-d'œuvre dans le cadre des activités économiques et commerciales contrôlées par les groupes blanc-métis arrivés dans la région. L'historiographie qui existe vis-à-vis de ce processus met l'accent sur l'inefficacité de ce décret. Or, l'analyse de la législation nationale et celle des documents se référant à l'embauche des équipages, permet d'affirmer qu'une partie de la population indigène qui travaillait dans le transport fluvial, tout comme autorités publiques de la préfecture ont essayé de concilier les droits constitutionnels de la population autochtone avec le développement économique régional.

Mots-clés : Bolivie, Amazonie, indigènes, législation, droits, main-d'oeuvre

\title{
The indigenous workforce in the fluvial trade of the Beni (Bolivia) in the second half of the $19^{\text {th }}$ century
}

\begin{abstract}
By decree of 6.08.1842 Beni's indigenous peoples acquired the category of citizens granting them property rights, equality and freedom. This also led to their incorporation into the workforce for economic and trade activities controlled by the white-mestizo groups who progressively moved to the region. The historiography emphasizes the ineffectiveness of the decree. In contrast, by analyzing the state's laws and the documents related to the hiring of crews, we can argue that part of the indigenous population dedicated itself to fluvial transport. The public authorities of the prefecture tried to reconcile the constitutional rights of the native population with the economic development in the region.
\end{abstract}

Key words: Bolivia, Amazon, indigenous, law, rights, workforce

En 1842 se creó el departamento del Beni sobre la mayor parte del territorio amazónico perteneciente a la república boliviana extendiéndose, por un lado, hacia los bosques tropicales septentrionales prácticamente desconocidos, surcados por infinidad de vías fluviales y poblados por comunidades no sometidas; y, por otro lado, hacia el centro-sur caracterizado por extensas sabanas tropicales sujetas a inundación y cuyos únicos núcleos de población eran las antiguas misiones de Mojos compuestas por distintas filiaciones étnicas que habían sido reducidas por los jesuitas en el siglo anterior.

Era el distrito más extenso y el menos poblado de toda la república, motivo por el que la concesión de tierras baldías, la expansión de la frontera agropecuaria y la extracción de la quina — primero- y la goma elástica — después - atrajeron una población blanco-mestiza que progresivamente se instaló con el control de las actividades económicas y comerciales. En consecuencia, el departamento beniano se organizó social y económicamente alrededor del acceso, la explotación y comercialización de los recursos naturales que ofrecía la región tal y como se plasmaría, años después, en su escudo departamental (fig. 1). En él fueron representadas gráficamente aquellas características fundamentales que simbolizaban el paisaje y los recursos naturales, cuya explotación permitió, en buena medida, el desarrollo del departamento del Beni y el enriquecimiento de sus grupos de poder durante el siglo XIX. 
Así, si en el cuartel central derecho se describía el bosque tropical con árboles de goma y en el cuartel central izquierdo aparecía la pampa ocupada con una res bovina y un corral; el cuartel superior lo ocupaba un cornucopia que, si bien en el escudo original de 1889 derramaba distintos frutos, el auge de la goma elástica haría que, posteriormente, vertiera libras esterlinas. A pesar de ello, nos interesa señalar el cuartel inferior donde fue representado un paisaje beniano singularizado por un caudaloso río a orillas del cual se alzaba una intrincada selva. Este río era surcado por una embarcación en el que, originalmente se destacaba una gran bandera boliviana en la popa que, años después, quedaría en un segundo plano al modificar la imagen para incluir

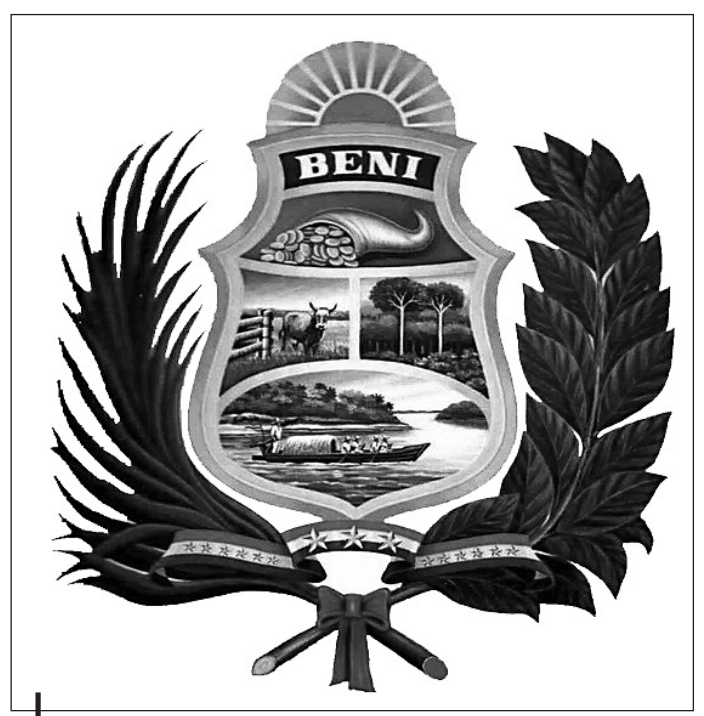

Figura 1 - Escudo actual del Beni una tripulación formada por nativos benianos caracterizados por sus camisetas y sombreros de paja, cuatro de ellos remando y uno ejerciendo de timonel desde la popa (Céspedes, 1942; Becerra Casanovas, 1992; Dávalos Mendoza, 2006).

La presencia de la población indígena en el escudo —únicas personas representadas en él- si bien es anecdótica también señala la importancia de la participación nativa en el proceso de construcción de la sociedad departamental como fuerza laboral activa en la tripulación de embarcaciones, una de las principales actividades comerciales del Beni. Elevados a la clase de ciudadanos, los indígenas que habían sido reducidos en misiones accedieron, a partir de 1842, a una libertad e igualdad en derechos que les incorporó a la economía regional como mano de obra de particulares y del mismo Estado. Para algunos autores, este nuevo estatus ciudadano no arrojó ningún resultado práctico para los indígenas, por la falta de continuidad y plena vigencia de las medidas de 1842 (Groff Greever, 1987: 171; Gamarra, 2010: 17-18). Para otros autores, en concreto para Lehm (1999: 39-40), la obtención de los derechos de libertad y propiedad prepararon el terreno para la expropiación de las tierras indígenas. Si bien coincidimos con lo propuesto por esta autora, al constatar que a inicios del siglo XX hubo una ocupación sistemática de terrenos de indígenas por parte de los grupos blanco-mestizos (Guiteras, 2010: 82-86; 2011b), en nuestra opinión, estos derechos y libertades también permitieron que, a lo largo de la segunda mitad del siglo XIX, una parte de la población indígena solicitara el acceso a la propiedad de la tierra (Guiteras: 2010; 2011a) y, en lo que interesa aquí, que algunos de los indígenas que ejercieron como mano de obra remera intentaran defender su libertad ante la connivencia de intereses públicos y privados de determinados grupos blanco-mestizos.

En consecuencia, a través del análisis de la legislación estatal que pretendía amparar los derechos de la sociedad indígena beniana y los documentos relativos a 
la contratación de tripulaciones indígenas hallados en distintos fondos bolivianos1, nos proponemos mostrar cómo algunos miembros de la población indígena que ejercía como tripulante de embarcaciones y algunas autoridades departamentales y estatales intentaron conciliar las garantías constitucionales de la población nativa beniana con el desarrollo económico de la región en la segunda mitad del siglo XIX. Por ello, en un primer apartado, se abordará las características principales de las medidas tomadas en 1842 a favor de los derechos de libertad, igualdad y propiedad indígena; en un segundo apartado, se señalaran los intentos de la prefectura para proteger la mano de obra indígena y evitar su uso particular y gratuito en el tráfico fluvial; en tercer lugar, se indagará sobre las prácticas de contratación de tripulantes; y, en cuarto lugar, se observará cómo, a fines del siglo XIX, el gobierno departamental se interesó por proteger la economía beniana lo que, al mismo tiempo, promovió la preservación de los derechos de los tripulantes ante la sobreexplotación de la que eran objeto.

\section{EL DECRETO DE 06.08.1842 Y LA POBLACIÓN INDÍGENA BENIANA}

El departamento del Beni surgió sobre un territorio ocupado básicamente por población indígena. Una parte de ella la componían distintas filiaciones étnicas no sometidas, dispersas en los bosques subandinos y tropicales del norte amazónico, percibidas como hostiles, bárbaras y salvajes, es decir, incivilizadas y cuyo número se desconocía. Otra parte de esta población estaba constituida por diversas filiaciones étnicas consideradas civilizadas por haber sido reducidas por los jesuitas en distintos núcleos de población y que, por aquel entonces, se hallaban bajo la tutela de curas seculares. Ya en 1830, D’Orbigny consignó en todas las misiones de Mojos un total de 23313 indígenas y 57 bolivianos blancos (D'Orbigny, 1846: 296-297), varones solteros, todos cruceños, en opinión de Moreno (1973 [1888]: 84). Este número se mantendría en los años siguientes, ascendiendo en 1839 a un total de 26763 indígenas reducidos2.

Fue en este último grupo en el que se fijaría el gobierno de José Ballivián (18411847), al observar que las garantías constitucionales dictadas «en la república en favor de los bolivianos no ha[bía]n sido extensivas a los desgraciados moradores» de la región³. Era necesario eliminar ciertas prácticas coloniales de tutelaje sobre

1 Para la elaboración de este artículo se ha trabajado con documentación del Archivo y Biblioteca Nacionales de Bolivia (ABNB), del Archivo Histórico de La Paz (ALP), del Museo de Historia de Santa Cruz (MHSC) y del Archivo de la Casa de la Cultura del Beni (ACCB). Este último, cabe mencionar, en el momento de nuestra revisión documental carecía de catalogación, motivo por el cual en el aparato crítico no aparecerán citadas las signaturas.

2 Ver D'Orbigny (1945 [1826-1833]: 1307, 1309, 1316, 1321, 1341, 1343, 1348, 1355, 1446) y «Estado General del número de almas que tiene esta dicha Provincia según los últimos censos que me han pasado los Curas en el presente año» (Trinidad, 26.22.1839) en MHSC, Provincias, Municipios y cantones, serie Mojos, Caja 1/25-82, f. 1.

3 Decreto de 06.08.1842, considerando n. ${ }^{\circ}$ 2, in Limpias Saucedo (2005 [1942]: 3). 
la población indígena ejercidas por los grupos económicos y comerciales que tenían contacto con estos grupos étnicos y las mismas autoridades públicas de la región. Ello implicaba luchar contra una percepción arraigada sobre los indígenas y que, tan solo unos años antes, en 1840, el gobernador Juan Bautista Antelo había verbalizado al señalar que la provincia estaba conformada por «naturales indígenas» los cuales «aún no están en estado de civilización ni pueden gozar de los derechos de ciudadanos»4.

Para ello se requirió el cambio de estatus de la región y de sus pobladores, de conformidad con los planteamientos modernizadores y el interés económico del área (Gamarra, 2010: 17). Por el decreto de 06.08.1842 y la instrucción de 08.08.1842, se independizaron los llanos de Mojos y el norte amazónico del departamento de Santa Cruz, quedando su población sujeta a las leyes vigentes, equiparándose al resto de la república. Entre los considerandos del decreto se afirmaba que los habitantes de las antiguas misiones «se hallan en un deplorable estado de esclavitud, de opresión y de miseria» dado que:

«El régimen gubernativo a que están sometidos los infelices indígenas de aquella provincia, es contrario a la naturaleza, a la ilustración, a los principios constitucionales proclamados por la república, y no puede continuar sin oprobio del gobierno que lo autorice»5.

Con el objetivo de adelantar económica, política y socialmente el área amazónica, por decreto de 06.08.1842 se elevó a todos los habitantes de Mojos a la clase de ciudadanos con todos los derechos de libertad, igualdad y propiedad que ella conllevaba y que se garantizaba a todos los bolivianos6; una ciudadanía que solo sería efectiva en aquellas poblaciones que habían sido reducidas y, por este motivo, capaces de ejercerla7. Cabe recordar aquí que si bien los reglamentos constitucionales señalaban que los ciudadanos bolivianos eran todos los habitantes de la república, de facto se diferenciaba entre aquellos que gozaban de los derechos civiles pero no de los derechos políticos —uso del poder electoral y obtención de empleos y cargos públicos- y aquellos otros que además de los derechos civiles ejercían también los políticos (Barragán \& Roca, 2005: 286). Por consiguiente, es claro que la ciudadanía dada a los indígenas benianos les confería, únicamente, derechos civiles a partir de los cuales podrían obtener la propiedad sobre la tierra y la libertad de comercio y de contratarse como mano de obra voluntariamente. En consecuencia, estos decretos dieron paso a las primeras regulaciones que involucraron a la población indígena (Gamarra, 2010: 17) compatibilizando los ideales perseguidos por los primeros gobiernos republicanos

4 «Carta del gobernador de Mojos a la Prefectura de Santa Cruz» (Trinidad, 12.01.1840) en MHSC, Provincias, Municipios y cantones, serie Mojos, caja 1/28-12, ff. 4-5.

5 Considerandos n. ${ }^{\circ} 1$ y 4 , in Limpias Saucedo (2005 [1942]: 3).

6 Artículo n. ${ }^{\circ}$ in Limpias Saucedo (2005 [1942]: 4) y artículo n. ${ }^{\circ} 2$ en Instrucción de 08.08.1842 en ALP, Sociedad Geográfica de La Paz (SGL), 1842, Caja 3, documento 33.

7 Quedaban, entonces, excluidos aquellos grupos nómadas y seminómadas, no sometidos, que eran percibidos como bárbaros y salvajes, en otras palabras, incivilizados. 
respecto a la realidad indígena amazónica con el objetivo de hacerla participar en la sociedad republicana.

En este sentido, las nuevas medidas eliminaron ciertas prácticas coloniales de tutelaje arraigadas y ejercidas por los empleados públicos y los pocos grupos económicos blanco-mestizos con los que tenía contacto la población nativa de la región. Una de las prácticas más extendidas eran los servicios gratuitos prestados al Estado que imponían la elaboración de determinada cantidad de productos tejidos, cultivos, carne, artesanías- con fechas fijas de entrega a los administrados del ramo (Block, 1997: 236-237); estos eran contrarios a los principios liberales proclamados por los gobiernos bolivianos, motivo por el cual por decreto del 6 de agosto de1842 se anularon8.

Sin embargo, en su lugar se estableció un sistema de trabajo que incluía tareas de carácter estatal como la construcción de caminos, la refacción de edificios, el acopio de madera y el transporte de carácter estatal por los ríos benianos 9 . Al mismo tiempo, la nueva situación jurídica de la población nativa permitió que esta participara activamente en la economía regional como mano de obra contratada por particulares en el servicio doméstico, las actividades agropecuarias y el tráfico fluvial (Limpias Saucedo, 2005 [1942]: 26-27). A pesar de ello, en lo que se refiere a la tripulación de embarcaciones, persistió la connivencia de los intereses públicos y privados en el uso de la mano de obra indígena, ignorando los derechos recién adquiridos por esta, actitud que, como veremos a continuación, algunas autoridades públicas intentaron subsanar.

\section{EN LA DEFENSA DE LA LIBERTAD DE LOS TRIPULANTES BENIANOS}

A lo largo de las décadas de 1840 y 1850 se incrementó la navegación de los ríos amazónicos. Primero, por el paulatino incremento de la población blanco-mestiza en la región resultado del aumento del número de empleados públicos10 y la llegada de grupos blanco-mestizos diversos a la región; y segundo, por la apertura del mercado beniano al interior de la república y al comercio internacional. Todo ello provocó un incremento del tráfico fluvial entre los departamentos de Cochabamba, Santa Cruz y el Beni, trasladándose correspondencia de oficio o particular, mercancías de todo tipo, pasajeros, autoridades públicas, etc. Su conducción a los núcleos urbanos benianos y a los principales centros de población

8 Unos días antes se había redactado una circular en la que señalaban los productos y en qué cantidad debían ser elaborados por los indígenas de la provincia, destacándose la manufactura textil, trabajos en madera, velas, jabón, carne y aceites. Véase Circular de 03.08.1842 in Limpias Saucedo (2005 [1942]: 5-6).

9 ABNB, Ministerio del Interior (MI), Prefectura del Beni, comunicaciones recibidas, tomo 101, n. ${ }^{\circ}$ 23, 03.11.1844, ff. 97-99 y 06.08.1844, s/f. Se estableció un calendario anual de tareas según los períodos de inundación, indicando la distribución por fechas de los indígenas en función de las manzanas del pueblo.

10 Por decreto de 21.04.1847 fueron designados los cargos públicos del departamento y sus sueldos. Disponible en: http://www.gacetaoficialdebolivia.gob.bo/normas/verGratis/36288 (revisado el 31.01.2011). 
bolivianos se llevaba a cabo a través de una marina mercante que recorría los ríos benianos y cuya conducción estaba a cargo de la población indígena (Block, 1997: 219).

A fines de la década de 1840, esta industria naval fue liberada, pudiéndose dedicar a ella todos los indígenas que quisieran hacerlo y entregándose embarcaciones a quienes fueran capaces de subordinar a toda una tripulación (Limpias Saucedo, 2005 [1942]: 44-45). En efecto, perpetuando la idea de Moreno (1973 [1888]: 21) que hacía de ese territorio «un país de remadores», los indígenas siguieron tripulando varias embarcaciones por la extensa red fluvial beniana. De este modo, los tripulantes nativos devinieron un elemento primordial para el desarrollo económico de la región ya sea por la incapacidad de los grupos blanco-mestizos de desempeñarse en la navegación de estos ríos o por las habilidades náuticas de los indígenas mojeños, cayubabas y canichanas, entre otros, que vivían a orillas del río Mamoré y sus afluentes.

Recién iniciada la vida independiente del Beni, este trabajo se ejercía gratuitamente si era por mandato estatal; por el contrario, si el beneficiario era un particular, empresario o comerciante, el trabajo debía ser debidamente compensado11. La existencia de dos formas en el uso de la mano de obra remera provocaría malentendidos para distinguir qué trabajos eran públicos y cuáles tenían un carácter particular, agravándose cuando la prefectura obligó a todos los comerciantes a realizar el transporte de correspondencia y artículos varios en sus propias embarcaciones ${ }^{12}$. Aseverada de los conflictos que podría ocasionar esta situación, desde el primer momento, la prefectura dictó órdenes y circulares a los distintos cantones del departamento con el objeto de tener un mayor control sobre el tráfico comercial desarrollado por la marina beniana y evitar el uso gratuito de la mano de obra indígena en beneficio de particulares. Quien mejor expresaría esta voluntad sería el prefecto Rafael de la Borda (1845-1848), al señalar que:

«Las ventajas de la industria y del comercio son absolutamente nulas sin el pleno ejercicio de la libertad. Está bien que el comerciante venda y compre, enganche tripulaciones para las canoas en que ha de extraer los productos de su jiro [sic], alquile obreros para las manufacturas que necesite y demás labores relativos a la especulación, pero hágase esto como se hace en todos los pueblos civilizados de la tierra»13.

En 1846 se tomaron las primeras medidas para regular la contratación indígena en el comercio fluvial, bien para el servicio privado de comerciantes o viajeros, o bien para el servicio estatal14; sin embargo, a tenor del contenido de las notas oficiales sabemos que a lo largo de las décadas de 1840 y 1850, su cumplimiento

11 Ver artículo n. ${ }^{\circ} 6$ de la Orden del 20 de abril de 1847 in Limpias Saucedo (2005 [1942]: 43-46).

12 ABNB, MI, Prefectura del Beni, comunicaciones recibidas, tomo 115, n. ${ }^{\circ} 37,19.02 .1846$, f. 47.

13 ACCB, «Circular reiterando la libertad indígena en el contrato» (Trinidad, 05.02.1846) [sin signatura]. El énfasis es del autor.

14 Ver «Cartas de la Gobernación de Mojos, guarnición de Mojos y departamento del Beni» (Trinidad, 8 de julio de 1846) en MHSC, Provincias, Municipios y cantones, serie Mojos, Caja 1/14-11, ff. 82-83 y 89-90. 
fue irregular. De este modo, al tiempo que el desarrollo económico y comercial de la región iba en aumento, las autoridades del Beni advertían que entre algunos comerciantes y empleados locales persistían prácticas calificadas de «intolerables», al impedir a los nativos que se desempeñaban conduciendo embarcaciones ejercer sus derechos y libertades otorgados mediante el decreto de 1842. Los prefectos, en primer lugar, señalaban que:

«Los naturales permanecen, como siempre, en la más degradante dependencia sobre lo que tanto les importa como es la libertad de manejarse en sus relaciones privadas, contratar, comercial y comprometerse» 15.

En segundo lugar, recordaban que:

«Los naturales [del Beni] son ciudadanos de la gran familia Boliviana y que se hallan exentos de prestar esa ciega obediencia que les exigiera el despotismo o la tiranía» 16 .

Y, finalmente, en tercer lugar, objetaban que esta situación:

«Es incompatible con el sistema de libertad bajo el que se halla constituida la provincia y, por otra, es doloroso ver ocupados a estos infelices sin gratificación alguna en viajes tan penosos como son expuestos a grandes riesgos»17.

Cabe señalar que en las décadas de 1840 y 1850 los tripulantes eran contratados por un mísero salario que, en muchas ocasiones, no llegaban a cobrar o, como pago, se les eximía de una parte de la contribución anual (Limpias Saucedo, 2005 [1942]: 35, 96, 111). Por entonces, la economía interna del departamento operaba, fundamentalmente sobre la base del trueque, comerciándose así muchos artículos de primera necesidad (Groff Greever, 1987: 44-45). En este sistema de trueque se incluían los salarios públicos que, a falta de líquido, se cobraban en especies (Limpias Saucedo, 2005 [1942]: 37, 66), ocurriendo lo mismo con la mano de obra indígena que, además de cultivar y producir los artículos de trueque, era pagada con los mismos. De ello dio cuenta el viajero norteamericano L. Gibbon quien, convencido que los nativos preferían ser compensados en tela, descubrió que «ellos sabían cómo hacer tela de algodón con sus propias manos» por lo que en el futuro, los indígenas le pedirían:

«Páguenos a nosotros y no a las autoridades, quienes se quedan con las monedas de plata y nos obligan a tomar la tela de algodón» (Gibbon, 1993 [1852]: 253-255, 288).

En efecto, lo señalado por los indígenas contratados por Gibbon y las circulares y normativas elaboradas desde la prefectura daban cuenta de una connivencia de intereses públicos y privados entre los grupos económicos y las autoridades locales. Desde la prefectura, por un lado, se atribuía a los comerciantes y a los

15 ACCB, «Circular reiterando la libertad indígena en el contrato» (Trinidad, 5 de febrero de 1846) [sin signatura]. El énfasis es del autor.

16 ACCB, «Circular denunciando las malas prácticas sobre la población nativa y ciudadana» (Trinidad, 4 de enero de 1855) [sin signatura]. El énfasis es del autor.

17 ABNB, MI, Prefectura del Beni, comunicaciones recibidas, tomo 107, n. ${ }^{\circ}$ 33, 30 de agosto de 1845 , f. 129. El énfasis es del autor. 
grupos económicos blanco-mestizos en general, una serie de vicios y falta de decoro que causaban desórdenes «perniciosos al orden y tranquilidad pública, y opuestos al sistema que se propone el Gobierno por la civilización de estos pueblos y en estos naturales» 18. Por otro lado, se lamentaba del papel que las autoridades públicas, particularmente locales, jugaban en el mercado laboral de la región «inter[viniendo] en los contratos y especulaciones particulares de los naturales de toda la provincia y en sus negocios privados y comerciales»19. En este sentido, la prefectura intentó romper ciertas relaciones de interés surgidas entre comerciantes y empleados locales quienes se aprovechaban de las tareas prestadas al Estado para emplear la fuerza laboral nativa en trabajos de carácter particular, sin contrato ni sueldo alguno20.

Si bien eran los corregidores quienes abusaban con más frecuencia de su poder, tal y como lo evidencian las continuas denuncias de su mala gestión en distintos ámbitos de la administración pública por parte de la prefectura21, cabe señalar que en la contratación de tripulantes intervenían también las autoridades indígenas: los caciques y los miembros del cabildo, en particular, los jefes de las parcialidades22. De ello dio cuenta el prefecto Carmelo María del Rivero (1855-1857) al señalar que ante la demanda de tripulantes por parte de algún comerciante, el corregidor lo transmitía a los caciques y estos a los jefes de las parcialidades, quienes:

«Nombran por consiguiente a los tripulantes sin averiguar si la esposa grávida o el hijo tierno necesitan de su auxilio, sin consultar si les place el pago que se ofrece, sin fijarse en que sus labores agrícolas demandan su precencia [sic] o si les afecta alguna dolencia que no les es permitido representar; y lo que es peor, más triste y doloroso, porque afecta a la moral pública, es que esos titulados jefes de parcialidad nombran precisamente al

18 Ver ACCB, «Carta del corregidor anunciando los desordenes cometidos por los comerciantes» (San Ignacio, 13 febrero de 1843) [sin signatura] y ABNB, MI, Prefectura del Beni, comunicaciones recibidas, tomo 134, n. ${ }^{\circ} 36,26$ de febrero de 1850, s/f.

19 ACCB, «Circular reiterando la libertad indígena en el contrato» (Trinidad, 5 de febrero de 1846) [sin signatura].

20 Ver ABNB, MI, Prefectura del Beni, comunicaciones recibidas, tomo 96, n. ${ }^{\circ}$ 48, 10 de octubre de 1843 y ACCB, «Circular denunciando las malas prácticas sobre la población nativa y ciudadana» (Trinidad, 4 de enero de 1855) [sin signatura].

21 En este sentido ver ABNB, MI, Prefectura del Beni, comunicaciones recibidas, tomo 96, n. ${ }^{\circ} 48,22$ de julio de 1843, s/f y 10 de octubre de 1843, s/f; tomo 143, n. ${ }^{\circ} 25,27$ de mayo de 1852, s/f; tomo, 216, n. ${ }^{\circ} 49,16$ de diciembre de 1883: f. 37; tomo 218, n. ${ }^{\circ} 42,27$ de noviembre de 1884: f. 60; tomo 228, n. ${ }^{\circ}$ 59, 1 de junio de 1886: ff. 17-18; tomo 244, n. ${ }^{\circ}$ 61, 23 de diciembre de 1889: f. 182.

22 El cabildo era la máxima institución gubernativa indígena creada bajo la administración jesuítica y que se mantuvo tras su expulsión. En época republicana el cabildo pasó a cumplir un rol de intercesión entre la comunidad indígena y el Estado, siendo su máxima autoridad el cacique, supeditado al corregidor, cargo usualmente ocupado por blanco-mestizos. Sujetos al cacique restaban los intendentes, los jefes de parcialidad o capitanes, los alcaldes, entre otros. El cabildo era integrado por la elite indígena denominada «familia» y compuesta por las parcialidades de oficio (asistentes religiosos, artesanos y guías de la política local), distinguiéndose del resto de la sociedad indígena conocida como «pueblo»; en consecuencia, se mantenía la dimensión jerárquica de la organización nativa legada por los jesuitas (Cortés, 1989: 42-43; Block, 1997: 79, 135-137, 235; Lehm, 1999: 28-32). 
esposo a cuya mujer virtuosa se trata de pervertir y al padre de la hija cuya inocencia se quiere corromper»23.

Para revertir la complicidad entre las autoridades locales, mayormente blancomestizas, y los empresarios de la región, a fines de la década de 1850, la prefectura redactó el primer reglamento de navegación para el Beni y eliminó la navegación fluvial de los servicios gratuitos que los indígenas prestaban al Estado. Por esta nueva medida se estipuló que todo contrato se efectuaría en presencia de las autoridades respectivas y que el número de remeros variaría según la capacidad de la embarcación. También fueron señaladas, por un lado, las obligaciones de los comerciantes y/o los pasajeros con los remeros a los que debían entregar comida y enseres para cubrir la travesía; y por otro lado, los derechos y deberes de los tripulantes en la conducción del cargamento hasta el punto de destino. Y finalmente, tras indicarse las rutas realizables (al interior de la república por el río Mamoré, hacia Santa Cruz por Guarayos, hacia Brasil y por el interior del departamento), se fijaron los sueldos que los tripulantes percibirían según el recorrido — entre 2 y 3 pesos 24 — señalándose que «desde el Estado, S. E. el Presidente para abajo, nadie absolutamente podrá exigir servicio gratuito a los naturales en la navegación» (art. transitorio 20) 25 . Aprobado en 1858, este reglamento entraría en vigor en la década de 1860 (Limpias Saucedo, 2005 [1942]: 147-148) y sentaría las bases para el desarrollo del tráfico comercial por los ríos benianos en las décadas siguientes.

\section{LOS TRIPULANTES BENIANOS EN EL COMERCIO FLUVIAL}

A lo largo de la segunda mitad del siglo XIX, las actividades económicas de la región fueron variadas. En las estribaciones andinas fue importante la recolección de cascarilla hasta la década de 1870, cuando disminuyó su demanda en el comercio internacional. Por aquel entonces, en el norte amazónico despuntaba otro producto, la goma elástica, cuya explotación aumentó a partir de la década de 1880 y se extendió hasta mediados del siglo XX. En el área pampeana, de los cultivos de algodón, azúcar y cacao y del carneo de ganado se elaboraban una gran diversidad de tejidos, aguardiente, carne, sebo y cuero, destinados al

23 ABNB, Ministerio de Hacienda (MH), Siglo XIX-Prefectura del Beni, tomo 141, n. ${ }^{\circ} 28,1$ de julio de 1855, s/f. En este sentido véase también ACCB, «Circular reiterando la libertad indígena en el contrato» (Trinidad, 5 de febrero de 1846) [sin signatura] donde se hacía énfasis a la «funesta intervención de caciques y corregidores» en la contratación, así como también la «Denuncia al cacique de Trinidad por el envío sin permiso del corregidor de una canoa a San Pedro de Caja» (Trinidad, 10 de enero de 1868) [sin signatura].

24 Se fijaron dos pesos desde Trinidad a los departamentos adyacentes, aumentando hasta un máximo de 3 pesos si se partía desde Reyes, San Borja y El Carmen. En la navegación al Brasil se pagaría la mitad de lo que se pagaría por subir. En la navegación interior el precio base sería de dos reales por desplazarse entre pueblos vecinos hasta cuatro reales para llegar a San Ignacio, Santa Ana y Exaltación. Recordemos que 8 reales equivalían a un peso.

25 Primer Reglamento de Navegación para el distrito del Beni de 01.07.1858 in Limpias Saucedo (2005 [1942]: 129-132). 
mercado interior y exterior (Dalence, 1851: 315-316; Limpias Saucedo, 2005 [1942]: 22, 26). La cascarilla se enviaba desde Apolo, Reyes o Rurrenabaque, río arriba hasta el final de la navegación en los Andes, desde donde se dirigía hasta los puertos del Pacífico (Fifer, 1970: 118; Block, 1997: 225). Hacia el Brasil se mandaban cargamentos de aceite de copaiba, sebo, pieles y goma elástica y se regresaba con té, canela, especias y pimienta (Keller \& Keller, 1870: 16; Limpias Saucedo, 2005 [1942]: 27, 59). De Santa Cruz y Cochabamba se importaba sal, harina, patatas y mercancías varias y se exportaba cacao y azúcar (Mathews, 1879: 152; Gibbon, 1993 [1852]: 267, 290).

La ubicación en la hoya amazónica del departamento beniano hizo que las principales y, en gran parte, únicas vías de comunicación fueran los ríos. De este modo, el tráfico fluvial propiciaría la expansión comercial departamental hacia áreas limítrofes, la ampliación de la frontera interna beniana con la llegada de nuevos colonos blanco-mestizos que avanzarían hacia el norte amazónico y el aumento de la demanda de mano de obra remera para desarrollar el intercambio comercial. En efecto, la indefinición de los límites departamentales y nacionales sobre este territorio dio lugar a la aparición, por parte de algunas autoridades públicas, de un discurso por el que las embarcaciones tripuladas por indígenas benianos eran el principal factor que permitiría «recobrar los derechos de la república a la navegación de nuestros ríos» (Limpias Saucedo, 2005 [1942]: 36). Esta percepción da cuenta de la importancia que a lo largo de la segunda mitad del siglo XIX adquirirían estos trabajadores. Efectivamente, durante todo el período estudiado aquí, los tripulantes benianos prácticamente monopolizaron el comercio regional remando periódicamente sus embarcaciones hacia el Brasil (Block, 1997: 228-229) y convirtiéndose en el principal elemento laboral de la región.

Las dificultades que entrañaba la navegación de los ríos amazónicos, las condiciones físicas y psíquicas que debían soportar los tripulantes durante las travesías y los efectos negativos de su alejamiento en la vida familiar, favorecieron que no pocos nativos se opusieran a participar en el comercio fluvial y/o requirieran de ciertas formalidades para realizarlo, como veremos a continuación.

Los distintos viajeros que, entre las décadas de 1850 y 1870, visitaron el Beni afirman que los indígenas no osaban desobedecer las órdenes que recibían de las autoridades locales (Keller \& Keller, 1870: 21; Keller, 1875: 81; Gibbon, 1993 [1852]: 296), quienes proponían, entre toda la población nativa, los tripulantes que fletarían las embarcaciones benianas. Si bien esto es así, existen evidencias que nos indican que, desde el primer momento, algunos indígenas intentaron evitar embarcarse tanto en el tráfico comercial desarrollado por particulares como en las travesías de carácter estatal. Los contemporáneos afirmaban que en las expediciones llevadas a cabo por José A. Palacios26 entre 1844 y 1847 «los hombres pasaron tan mal rato en aquella

26 Palacios fue uno de los primeros nacionales que, designado por el gobierno boliviano, exploró, recorrió y navegó las principales vías fluviales del departamento del Beni, a la búsqueda de sus confluencias, su navegabilidad — siendo el primero en superar los rápidos que, posteriormente, serían conocidos como Cachuela Esperanza- y su vinculación con el río Amazonas y el océano Atlántico (Palacios, 1944 [1844-1847]). 
ocasión que, cuando regresaron y contaron a sus familias y vecinos, dejaron tal impresión que se negaron a ir nuevamente y abandonaron las canoas» (Gibbon, 1993 [1852]: 293). En este sentido, sabemos de indígenas que cuando las autoridades locales les propusieron ejercer como tripulantes se negaron a embarcarse. Estos alegarían ser «desconocida por ellos la navegación del río Madera», temiendo ser víctimas de «aquellos lugares tan insanos» y carecer de armas para «defenderse de las tribus salvajes»»27, cuyos ataques a las canoas que surcaban los ríos amazónicos eran frecuentes28. Además, los indígenas se mostraban reacios a abandonar sus familias y sus chacos, considerando un mes tiempo estimado en ir y volver de Santa Cruz o Cochabamba, un viaje largo 29 y «prefiriendo muchos quedarse y recoger su cosecha, que ir de viaje» (Gibbon, 1993 [1852]: 331). Como señala Roca (2001: 99-100), conforme avanzaría el siglo XIX, la demanda cada vez más masiva de tripulantes ocasionaría una fuerte desestructuración familiar y comunitaria por permanecer largo tiempo fuera de su hogar, no serles permitidos llevar sus mujeres con ellos, descuidar sus siembras, lo que conllevaría un desabastecimiento alimenticio30. Esto favoreció que, a partir de la década de 1880, algunos de los indígenas alegaran, simplemente, no ser «su voluntad» marchar hacia el Madera con el empresario que pretendía contratarlos31.

A todo ello cabe agregar, además, las condiciones en las que se desarrollaba la navegación que, en algunas ocasiones, fue denunciada por los remeros indígenas con el objetivo de salvaguardar, ante las autoridades y los grupos locales y regionales, sus libertades civiles. Según distintos coetáneos, durante estos viajes era usual azotar a los tripulantes cuando se retrasaban en el viaje (Gibbon, 1993 [1852]: 288, 296), no proporcionarles víveres suficientes (Keller \& Keller, 1870: 5) y obligarles a cargar sobre sus lomos mercancías, equipajes y pasajeros hasta los puertos de embarque como bestias de carga (Limpias Saucedo, 2005 [1942]: $35-36,111)$. Se toma como ejemplo una expedición en la que los tripulantes aseguraron haber sido víctimas de diversos malos tratos por parte del patrón de la embarcación 32 y que, en nuestra opinión sintetiza, por un lado, las actitudes que los comerciantes tenían durante el transporte de mercancías por los ríos amazónicos y su complicidad con los empleados locales; y, por otro lado, muestra cómo algunos indígenas conocían y usaban los mecanismos que ofrecía la justicia para denunciar el abuso de sus derechos.

27 ACCB, «Los naturales desisten de emprender la navegación al Madera» (San Joaquín, 19 de noviembre de 1846) [sin signatura].

${ }^{28}$ ACCB, «Ataque de los salvajes a tripulaciones» (Huacaraje, 15 de marzo de 1868) [sin signatura].

29 «Los indios llevaban la cuenta del número de días lejos de sus esposas haciendo un pequeño corte en el mango de sus remos cada séptimo día y se piensa que una tripulación que regresa con más de cuatro cortes ha estado ausente un largo tiempo de Trinidad» (Gibbon, 1993 [1852]: 293-294).

30 Un contemporáneo señalaría «que sucede todavía, que el regreso después de un mes encuentra el padre y el esposo perdida la sementera [y] la casa convertida en morada de desolación» en ABNB, MH, Siglo XIX-Prefectura del Beni, tomo 141, n. ${ }^{\circ} 28,1$ de julio de 1855, s/f.

31 ACCB, «Indígenas se niegan a ser contratados» (Trinidad, 9-10 de junio de 1883) [sin signatura].

$32 \mathrm{ACCB}$, «Indagatoria para esclarecer los hechos de que asegura han sido víctima [sic] la mayor parte de los tripulantes que condujeron al ciudadano David Cronenbold a la provincia del Pará» (Trinidad, 1 de septiembre de $1866-5$ de enero de 1867) [sin signatura]. 
En 1866 el comerciante David Cronenbold33 fletó una expedición y, como era usual, contrató los servicios de grupos étnicos ribereños, en este caso mojeños, movimas, cayubabas y canichanas con los que armó siete embarcaciones para viajar al Pará, en Brasil34. Según los testimonios de la indagatoria, iniciada ese mismo año, Cronenbold «solo quería que todo el día estuviesen en un continuo trabajo del remo» y ni siquiera les «dejaba bajar a descansar y [le]s tenía a todos sentados en [su]s puestos», llegando al punto que «padecían de víveres porque no se les daba» y les habían quitado «todas [su]s herramientas, hamacas y calijas [sic], que por las noches no tenía[n] con qué abrigar[se]»35. Tales fueron los maltratos de los cuales los indígenas afirmaron haber sido víctimas, con pistola en mano, de «palos, puñetes, garrotazos y patadas» llegando a ser flagelados «por capricho» con entre 50 y 500 azotes cuando heridos, hambrientos y agotados eran incapaces de remar tan fuerte y rápido como se les exigía. La respuesta a esta situación fue la huída de hasta cinco de las siete tripulaciones que, a pesar de ser perseguidas durante un tiempo, no fueron encontradas; no era una actitud insólita dado que el abandono de viajeros y comerciantes era una tónica en la navegación del río Madera (Mathews, 1879: 34 35; Gibbon, 1993 [1852]: 293; Limpias Saucedo, 2005 [1942]: 149) llegando en algunos casos hasta el asesinato de algunos patrones (Keller \& Keller, 1870: 14-15).

Al quedarse sin fuerza laboral suficiente para conducir el cargamento transportado, Cronenbold fue a la búsqueda de nuevos brazos benianos no sin antes desviarse hacia la fortaleza brasileña de Príncipe de Beira, donde dejó a los tripulantes enfermos para, con aquellos que estaban sanos, dirigirse hacia el cantón de San Joaquín, ya en territorio boliviano. Fueron estos indígenas regresados quienes, ante las autoridades públicas, expusieron y denunciaron todas las vicisitudes vividas durante el viaje. En su relato afirmaron que quienes se habían quedado en Príncipe de Beira se encontraban tan débiles que el «patrón no los dejaba que viniesen», en su opinión, por el «temor [de Cronenbold] de las autoridades el que tomasen medidas contra él por los bejámenes [sic] i ultrajes que hacía a la gente de su tripulación»36.

En este sentido, los tripulantes regresados señalaban que aquellos que quedaron en la fortaleza brasileña estaban fuera de la jurisdicción beniana y, por ende,

33 David Cronenbold era un comerciante alemán llegado a Bolivia desde el imperio del Brasil a inicios de la década de 1860. Dedicado inicialmente al comercio con Paraguay, con la guerra de la Triple Alianza (1864-1870) volcó todos sus intereses comerciales con Brasil, país del que llegó, en 1882 a ser vicecónsul. Cronenbold fue, asimismo, un importante armador de embarcaciones cuando la extracción de la goma elástica estaba en su apogeo y gran cantidad de lanchas y vapores surcaban los ríos amazónicos. De él se dice que también fue boticario, fundidor y arquitecto, además de propietario, agricultor e industrial. Murió en 1902 (Hollweg, 1995: 78-83).

34 Desconocemos el número de indígenas que conformaban toda la tripulación, sin embargo, Franz Keller (1875: 42) afirma que fletó siete canoas con 80 indígenas remeros, lo que nos da una idea aproximada de los que pudieron viajar con Cronenbold.

35 ACCB, «Indagatoria...», ff. 2v, 3, 4, 6, 6v, 7, 7v, 8v, 17.

36 ACCB, «Indagatoria....», f. 5v, 13v. Un testigo blanco-mestizo afirmaría que, a su paso por la fortaleza, los habría visto a orillas del río «sumamente enfermos, [h]inchados de los pies y de la cara i del cuerpo enteramente extenuado, las nalgas despedazas por los azotes» (ACCB, «Indagatoria....», f. 10). 
permanecían bajo la autoridad del comerciante sin recibir pago alguno por su trabajo37. Por el contrario, todos aquellos que sí volvieron al Beni, vieron fenecido su contrato como tripulantes y su vinculación con el patrón, siendo el momento de cobrar por ello. Por aquel entonces ya había entrado en vigor el reglamento de navegación beniano donde se establecían los sueldos de cada tripulante, en función del destino y el tiempo invertido (Limpias Saucedo, 2005 [1942]: 147 148) debiendo todos los patrones atenerse a él. Sin embargo, contraviniendo todo lo señalado en esta normativa, Cronenbold pagó a sus tripulantes con solo 6 pesos por los nueve meses que habían estado de viaje «sin dar[les] chancelo [sic] total de todos estos meses»38. Además, cabía agregar los trabajos que habían llevado a cabo en Villa Bella, sin remuneración alguna por la complicidad entre el comerciante y los empleados públicos de la población. Ignorando una vez más los derechos de la sociedad indígena, se confundieron los servicios públicos que debían prestar al Estado con los trabajos y las tareas particulares39. Como afirmaría uno de los indígenas:

«[el patrón] vendió nuestro trabajo como si fuéramos esclavos, sin darnos lugar a que por sí trabajásemos para nosotros mismos» 40 .

A tenor de estas aseveraciones que dan cuenta del alcance de las relaciones entre empresarios y empleados públicos locales señaladas en el punto anterior, fueron múltiples los atropellos perpetrados por este comerciante. A pesar de ello, la existencia de un proceso judicial que pretendía esclarecer las denuncias hechas por los tripulantes indígenas contra David Cronenbold nos parece muy relevante. En nuestra opinión, en primer lugar, indica el interés mostrado por los empleados de la prefectura por resolver lo ocurrido, mantener el orden público en base a los principios liberales expuestos en las normas y circulares que hemos señalado en el punto anterior y evitar la consabida connivencia entre intereses públicos y privados; si bien desconocemos cuál fue el resultado de estas indagaciones, sabemos que Cronenbold siguió su actividad empresarial con Brasil (Hollweg, 1995: 80-82). En segundo lugar, muestra que las relaciones entre los grupos blanco-mestizos y los grupos indígenas no fueron ni uniformes ni siempre de enfrentamiento; en realidad, las actitudes y prácticas de ambos grupos fueron diferentes según las circunstancias e, igualmente, no todas las autoridades públicas actuaron en forma similar ni tuvieron los mismos intereses. En cualquier caso, lo dicho hasta ahora ejemplifica bien la voluntad prefectural por evitar el abuso de poder por parte de las autoridades locales. Y, en tercer lugar, revela el conocimiento de la población nativa

37 ACCB, «Indagatoria...», f. 19v. Véase también «Razón demostrativa formada por el Corregidor que suscribe del número de naturales que cada vecinio tiene en concierto...» (San Ignacio, 27 de agosto de 1866) en ALP, SGL, 1866, Caja 3, doc. 67, donde se señala que de la tripulación fletada por Cronenbold aún restan siete indígenas por regresar.

38 ACCB, «Indagatoria...», f. 16v. Franz Keller (1875: 78-79) también daría cuenta del poco salario cobrado por los tripulantes a pesar de las inclemencias a las que se exponían.

39 Este no fue el único caso que conocemos de connivencia entre lo público y lo privado contra el que los indígenas lucharon llegando incluso, en San Ignacio, a asesinar al corregidor (Gibbon, 1993 [1852]: 298; Limpias Saucedo, 2005 [1942]: 108).

40 ACCB, «Indagatoria...», f. 18. 
de los derechos civiles, prácticas y mecanismos establecidos por la administración boliviana para defenderlos; las denuncias efectuadas por estos indígenas remeros y las negativas a embarcarse, particularmente si los viajes se consideraban demasiado largos, nos informan de la voluntad de esta mano de obra por intentar amparar sus libertades en su participación de la vida socioeconómica beniana.

Antes de cerrar este apartado, cabe señalar que ninguno de los testigos nativos llamados a nombrar su profesión lo hizo como «tripulante» sino como sacristanes, herreros, músicos y labradores; ello podría indicar que no se trataba de un oficio fijo ejercido siempre por unos mismos individuos, sino de un trabajo periódicamente asignado a los indígenas varones ribereños que únicamente ellos sabían prestar y que, según algunos casos, habrían preferido no realizar. A pesar de ello, como veremos a continuación, la importancia que adquirirían sus servicios al avanzar el siglo XIX propiciaría la aparición de nuevas normativas de contratación que, si bien pretendían favorecer el desarrollo económico departamental, permitieron salvaguardar los derechos y libertades de los indígenas tripulantes.

\section{EL ESTADO BOLIVIANO Y LAS LIBERTADES DE LOS TRIPULANTES INDÍGENAS}

Como ya hemos señalado, a partir de la década de 1870 la explotación de la cascarilla declinó, al tiempo que hubo incremento progresivo de la explotación de la goma elástica en toda la Amazonía por la alta demanda de productos elaborados en látex en el mercado internacional (Gamarra, 2007). La importancia que adquirió esta nueva actividad económica potenció la llegada de diversos emprendedores que, por un lado, se internaron en los bosques amazónicos descubriendo nuevas zonas con árboles de la goma y, por el otro lado, obtuvieron derechos de libre explotación y comercialización de este producto. Ello dio lugar a un gran trasvase de mano de obra en los bosques y un aumento considerable del número de embarcaciones que surcaban los ríos hacia el océano Atlántico lo que, al mismo tiempo, supuso un incremento de la demanda de indígenas ribereños, por ser considerados, gracias a sus habilidades náuticas, los mejor cualificados para llevar a cabo estas travesías. Esta situación motivó una importante disminución de la población nativa y el desarrollo por parte de las autoridades departamentales y estatales de distintas políticas tendentes a preservar el acceso a la mano de obra y proteger, en concreto, las libertades de los indígenas dedicados a la navegación.

Las actividades extractivas motivaron, por un lado, un aumento en el comercio de la hoya amazónica y, por otro lado, el traslado de un gran contingente de mano de obra, cuyas condiciones de vida y laborales derivaron en un progresivo descenso de la fuerza laboral del Beni. No en vano, este coincide con la llegada, cada vez más significativa en número, de grupos blanco-mestizos al Beni que empezaron a desarrollar distintas actividades económicas vinculadas a la explotación de los recursos naturales. En efecto, a la luz de los datos que ofrece la figura 2 se puede observar que la población indígena se mantuvo relativamente estable hasta fines 


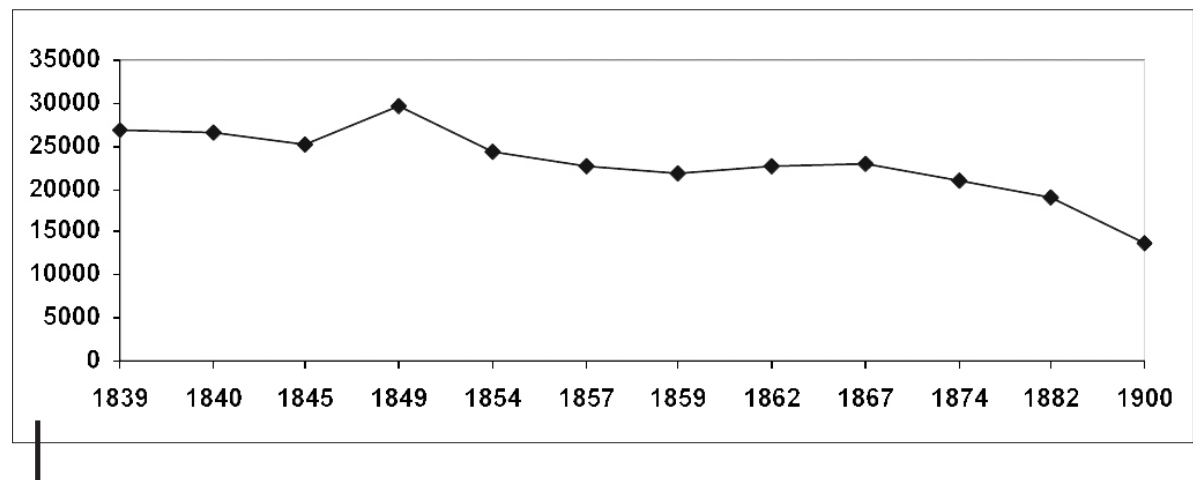

Figura 2 - Demografía indígena del Beni, 1839-1900

Elaboración propia en base a Block (1997: 231) con datos de ALP, SGL, Caja 3, docs. 34, 37, 38, 40, 41, 42 y 45; ABNB, TNC-Revisitas, Beni, 1849, 1854, 1857, 1859, 1862, 1867 y 1872-74

de la década de 1860 cuando se inició un leve descenso que a fines del siglo XIX se agudizaría.

El declive demográfico indígena fue documentado por autores contemporáneos que hicieron especial énfasis en su impacto sobre los nativos que trabajaban navegando los ríos amazónicos. Los viajeros se sorprendieron de la escasa población en núcleos como Exaltación: mientras sus pobladores indígenas habían sido los tripulantes más apreciados en épocas anteriores, en la década de 1870 ya no había suficientes hombres para fletar embarcaciones (Keller \& Keller, 1870: 1821; Keller, 1875: 82; Mathews, 1879: 123, 131, 135). Según Mathews, en 1873 habían salido al río Madera 43 embarcaciones de las que solo unas 13 regresaron; en su opinión, esto significaba que unos 300 indígenas se habían quedado en el camino. Asimismo, según sus cálculos, entre 1862 y 1872 el Beni había perdido cada año un promedio de mil hombres (Mathews, 1879: 132). Este descenso respondía a los muchos tripulantes que habrían sucumbido en los rápidos de los ríos, las fiebres, el agotamiento y los malos tratos (Limpias Saucedo, 2005 [1942]: 174). De hecho, Roux (2000: 185) estima las pérdidas humanas en el transporte fluvial en alrededor del $5 \%$ del total de la tripulación, dándose especialmente en el remonte del río. A ello, cabía agregar aquellos nativos que quedaron en los gomales o en establecimientos diversos (Limpias Saucedo, 2005 [1942]: 79) impidiéndoseles o retrasando de motu proprio el regreso al hogar41.

La fuerza laboral indígena era vital para la economía beniana ejerciendo como siringueros, peones, vaqueros, remeros, etc., por lo que su descenso poblacional afectaba directamente a la economía departamental. No obstante, como se ha señalado, el avance económico del Beni estaba estrechamente vinculado a la

41 Ver MHSC, Prefectura del Beni, Caja 2/44-08, 12 de marzo de 1850, f. 27 y ABNB, MI, Prefectura del Beni, comunicaciones recibidas, tomo 200, n. ${ }^{\circ} 22,12$ de febrero de 1874-12 de marzo de 1874, ff. 9-13; tomo 211, n. ${ }^{\circ} 18,6$ de mayo de 1881 y 1 de julio de 1881, ff. 1-2. 
explotación de los recursos naturales de la Amazonía boliviana, destinados exclusivamente al mercado externo. Sin embargo, el comercio beniano dependía directamente del tráfico fluvial convirtiendo así a los indígenas que tripulaban las embarcaciones en los puntales de la economía beniana y a los que se debía proteger, diferenciándoles, en buena medida, del resto de población indígena.

En este sentido, la prefectura beniana — primero-y los gobiernos bolivianos - después - promovieron una serie de normativas relativas al trabajo de los remeros indígenas con el objeto de proteger el desarrollo económico de la Amazonía, al tiempo que, revalidaban las libertades y derechos civiles adquiridos en 1842. Ya en la década de 1860 se había restringido la movilidad de la población sin pasaporte o licencia, fue prohibido el cambio de patrón sin consentimiento del mismo trabajador y la autoridad correspondiente y se limitó la duración de los contratos $^{42}$. Sin embargo, la persistencia de la connivencia entre comerciantes y autoridades locales, las denuncias y las negativas a embarcarse por parte de algunos nativos, provocaron que se amparara, cada vez más, el trabajo, la vida y las condiciones de trabajo de los tripulantes. De este modo, en la década de 1870, desde el gobierno departamental se fijó en 10 bs. la fianza que debían pagar los patrones para utilizar los servicios del personal remero y se señaló que aquellos que viajaran hasta Brasil quedarían bajo la protección del Vicecónsul de Bolivia en ese país43. También se aumentó el salario de los tripulantes pasando de 2 a 5 pesos mensuales, cantidad que se incrementaría en un 30-40\% a fines de la misma década; de este modo llegaron a cobrar 8 pesos de ida hasta los departamentos adyacentes y 2 más de vuelta cuando, dos años antes se cobraba entre 6 y 7 pesos por ir y volver al mismo lugar (Mathews, 1879: 161). No en vano, al ingresar en la década de 1880, un coetáneo afirmaría que los «tripulantes de las embarcaciones [...] son bien pagados», al contrario de los indígenas que se dedicaban a otras tareas (Ministerio de Hacienda, 1882: 12).

Sin embargo, cabe señalar que la mejora de las condiciones laborales y salariales no respondió tanto al interés por solventar las denuncias de las condiciones en la navegación que impulsaban a los indígenas a negarse a embarcar, sino a la necesidad de preservar la mano de obra en la región que no se sintiera tentada por otros negocios agropecuarios, gomeros y forestales que, a fines del siglo XIX, estaban enriqueciendo buena parte de la sociedad blanco-mestiza que se había desplazado al Beni. El personaje que mejor ejemplifica este pensamiento fue el prefecto Mamerto Oyola, quien afirmaría:

«Tendría muy corta vista quien no viese, en el despueble, la total ruina del comercio interior y exterior, industria y agricultura de estos pueblos. Si son conducidos al Madera iqué brazos trasportarán las producciones al interior de la República? ¿Quiénes serán los remeros para el comercio con el Departamento de Santa Cruz? ¿Se preferirá la muerte de estas poblaciones,

\footnotetext{
42 Orden de 03.02.1861 in Limpias Saucedo (2005 [1942]: 140-141).

43 ABNB, MI, Prefectura del Beni, comunicaciones recibidas, tomo 192, n. ${ }^{\circ} 26 a, 19$ de julio de 1870, f. 28; $\mathrm{MH}$, Siglo XIX-Prefectura del Beni, tomo 182, n. ${ }^{\circ} 31,1871$.
} 
por dar vida a la industria gomera? ¿Se preferirá intereses particulares a intereses de todo un pueblo? [...] Lo que se quiere, es evitar la muerte de estas poblaciones. Nadie se opone a que marche el indio de Chiquitos o Cordillera, porque esos indios no son remeros que atienden el comercio» (Oyola, 1883: 2).

En efecto, desde mediados de la década de 1880 la economía amazónica experimentó un gran auge provocando una intensificación del intercambio comercial local, regional e internacional; el tráfico de personas y mercaderías se hacía en muchos casos en transporte fluvial, a remo. La sobreexplotación de la fuerza de trabajo utilizada provocó un aumento significativo de la mortalidad de tripulantes y la consiguiente disminución de los brazos que tal actividad exigía 44 (Ministerio de Hacienda, 1882: 2-8; Arze, 1890: 27; Rivas, 1896: 2-5). Esta situación motivó que el Estado boliviano decidiera intervenir con la promulgación de la ley de 24.11.1883 en la que se señalaron distintas medidas de protección a los indígenas45. En general, se abolieron todos los aranceles y reglamentos específicos que existían sobre la población nativa beniana. Y, en concreto, se indicó cómo debería realizarse toda contratación de peones en el futuro, restringiéndose su movilidad hacia los departamentos adyacentes y, en especial, hacia el río Madera. De este modo, «respecto a la garantía constitucional de la libertad de locomoción» se indicó que los indígenas podrían «libre y espontáneamente» celebrar contratos para trasladarse a otros lugares, en especial, a Brasil. En este sentido, se estipuló el tiempo máximo que podrían estar de viaje y que el salario sería cobrado en moneda, se obligó a distintas autoridades a llevar una matrícula de todos los tripulantes embarcados para comprobar su regreso y se estableció una fianza sobre cada remero que, en caso de no regresar, debería ser entregada a la familia (arts. $5^{\circ}$ a $9^{\circ}$ ). Esta fianza iría creciendo conforme finalizaba el siglo XIX; situada en 10 bs. en 1870, una década después se situaría entre los 50 y 100 bs. (Oyola, 1883: 2; Anuario, 1884: 286) llegando hasta los 300 bs. en 189546.

Al ingresar al siglo XX, el transporte fluvial se modificó levemente con la aparición de las primeras lanchas a vapor adquiridas por diversos empresarios regionales que, además de efectuar un transporte particular, también deberían prestar servicios gratuitos cuando fuera requerida una conducción de carácter estatal (Arze, 1890: 29; Rivas, 1896: 2-4; López, 1908: 11-12, 15-16; Vaca Chávez, 1913: 31). A pesar de todo, la existencia de un número elevado de embarcaciones a remo en todo el departamento mantuvo la demanda de tripulantes indígenas $y$, con ello, la necesidad de salvaguardar la fuerza laboral nativa hasta el primer tercio del siglo XX. En efecto, tras la aprobación, por decreto del 30 de enero de 1912, de un nuevo reglamento de navegación

44 ABNB, MI, Prefectura del Beni, comunicaciones recibidas, tomo 254, n. ${ }^{\circ} 70,4$ de junio de 1890, s/f; tomo 271, n. $^{\circ} 75,2$ de julio de 1893, ff. 74-75.

45 Ley del 24 de noviembre de 1883 in Anuario (1884: 285-288); el proyecto de dicha ley del 7 de octubre de 1882 in Oyola (1883: 24-30).

46 ACCB, Copiador de Oficios, 1895-1896, 24 de julio de 1895, f. 40. 
para toda la república, la Cámara de Comercio de Trinidad aún haría hincapié en la idiosincrasia de la navegación beniana y la necesidad de garantizar la ida y regreso de los tripulantes, así como sus derechos y obligaciones en el desarrollo de sus tareas, para preservar el comercio fluvial del departamento tal y como se había desarrollado hasta el momento47.

\section{REFLEXIONES FINALES}

«Llegaban los precursores de la nueva industria y fundaban poblaciones y asentaban sus trabajos en plena selva, hasta donde llegó la soberanía de la patria en los linderos del Perú y del Brasil [...] iQuién, navegando hacia rumbos desconocidos, hubiera impulsado el remo que llevó las naves cargadas con el precioso producto, a lo largo del río Beni, del Mamoré, el Madera y el Amazonas, desafiando las cachuelas, hasta tocar con los puertos donde se troca [sic] la goma en el oro que dan los mercados ultramarinos!» (Limpias Saucedo, 2005 [1942]: 209).

Así se expresaba, en la década de 1920, el autor beniano Manuel Limpias Saucedo para señalar la importancia que tuvieron aquellos indígenas que, a remo, surcaron los muchos ríos que atravesaban el departamento del Beni participando del progreso de la economía regional. En efecto, a lo largo de la segunda mitad del siglo XIX la incorporación del Beni a la república boliviana y de esta al mercado internacional fue posible gracias al tráfico fluvial que se desarrolló en la hoya amazónica y favoreció el traslado de personas, mercancías e ideas. Quienes lo hicieron factible fueron numerosos indígenas de distintas filiaciones étnicas, particularmente mojeños, canichanas, cayubabas y movimas, que habían sido reducidos en las misiones y que, dadas sus habilidades náuticas, fueron requeridos para navegar los caudalosos ríos de la región.

Los derechos de igualdad y libertad que la población indígena beniana adquirió por el decreto del 6 de agosto de 1842 dieron lugar a la incorporación de la población indígena, como mano de obra, a las actividades económicas desarrolladas por los grupos blanco-mestizos desplazados al Beni. A pesar de ello, a lo largo del siglo XIX persistió la complicidad entre estos grupos económicos y las autoridades públicas locales, particularmente los corregidores, que provocó que estos derechos fueran frecuentemente menospreciados, dando lugar a una connivencia de intereses públicos y privados. Centrándonos en la mano de obra tripulante, las fuentes señalan el interés mostrado por la prefectura beniana en evitar la persistencia de estas prácticas, bien expulsando las autoridades locales de sus cargos o bien reforzando el control sobre la contratación de la mano de obra por parte de particulares. Así, entre las décadas de 1840 y 1860, se elaboraron y aplicaron circulares y normativas varias que pretendían mantener los principios

47 Véase el reglamento de navegación disponible en: http://www.gacetaoficialdebolivia.gob.bo/normas/ verGratis/40969 (revisado el 30 de abril de 2011); véase también el periódico La Democracia, «Cámara de Comercio» (Trinidad, n. ${ }^{\circ} 489,25$ de enero de 1913) 
liberales republicanos para, a partir del 1870, procurar conciliar las garantías constitucionales de los indígenas remeros con el desarrollo económico que estaba viviendo la región. Si bien el decreto de 1842 y las distintas reglamentaciones que le sucedieron no pretendían tanto favorecer las condiciones laborales y vitales de los tripulantes como asegurar el mantenimiento de una efectiva marina mercante en la región, su existencia favoreció que los nativos lejos de mostrarse pasivos, intentaran aprovecharse de estas normativas, negándose a tripular embarcaciones si no era su voluntad y verbalizando su descontento ante el menoscabado de sus libertades. De este modo, al contrario del resto de la mano de obra nativa que se dedicaba a la explotación agropecuaria, gomera y forestal, a lo largo del siglo XIX, los tripulantes indígenas aprovecharon que su fuerza laboral era altamente demandada por la economía departamental; al mismo tiempo utilizaron prácticas y mecanismos legales para, en la medida de lo posible, ejercer sus derechos en el seno de la sociedad republicana.

\section{Referencias citadas}

\section{Fuentes primarias}

Archivo y Biblioteca Nacionales de Bolivia (ABNB)

Ministerio del Interior (MI)

Ministerio de Hacienda (MH)

Tribunal Nacional de Cuentas (TNC)

Archivo Histórico de La Paz (ALP)

Sociedad Geográfica de La Paz (SGL)

Museo de Historia de Santa Cruz (MHSC)

Provincias, Municipios y cantones

Prefectura del Beni

Archivo Casa de la Cultura (ACCB)

\section{Fuentes impresas}

ANUARIO, 1884 - Anuario de Leyes y Supremas Disposiciones de 1883, 302 pp.; La Paz: Imprenta de El Comercio.

ARZE, E., 1890 - Informe de la Prefectura del Beni en el año 1890, 42 pp.; Trinidad: Imprenta de Francisco Suárez \& Hnos.

D'ORBIGNY, A., 1846 - Descripción geográfica, histórica y estadística de Bolivia, Tomo I, 402 pp.; París: Librería de los Señores Gide y Compañía.

D'ORBIGNY, A., 1945 [1826-1833] - Viaje a la América Meridional, 4 tomos, 1614 pp.; Buenos Aires: Editorial Futuro.

DALENCE, J. M., 1851 - Bosquejo estadístico de Bolivia, 391 pp.; Chuquisaca: Imprenta de Sucre.

GIBBON, L., 1993 [1852] - Exploración del Valle del Amazonas, Tomo II, 448 pp.; lquitos: Centro de Estudios Teológicos de la Amazonía, Abya Yala. 
KELLER, F., 1875 - The Amazon and Madeira Rivers. Sketches and descriptions from the note-book of and explorer, 242 pp.; Philadelphia: J. B. Lippincott and Co.

KELLER, J. \& KELLER, F., 1870 - Exploración del río Madera en la parte comprendida entre la Cachuela de San Antonio y la embocadura del Mamoré, 72 pp.; La Paz: Imprenta de la Unión Americana.

LÓPEZ, C., 1908 - Informe que eleva el Señor [...], Prefecto y Comandante General del Departamento del Beni, 155 pp.; Trinidad: sin pie de imprenta.

MATHEWS, E. D., 1879 - Up the Amazon and Madeira Rivers through Bolivia and Peru, 406 pp.; London: Sampson Low, Marston, Searle \& Rivington.

MINISTERIO DE HACIENDA, 1882 - Informes y documentos referentes al departamento del Beni, 32 pp.; La Paz: Imprenta de La Libertad.

MORENO, G. R., 1973 [1888] - Catálogo del Archivo de Mojos y Chiquitos, 583 pp.; La Paz: Ed. Juventud.

OYOLA, M., 1883 - Informe que eleva ante el Supremo Gobierno el prefecto del Departamento del Beni, 30 pp.; La Paz: Imprenta de La Industria.

PALACIOS, J. A., 1944 [1844-1847] - Exploraciones, 77 pp.; La Paz: Editorial del Estado.

RIVAS, C., 1896 - Informe administrativo del Prefecto y Comandante Ceneral del Departamento del Beni, 24 pp.; Trinidad: Tipografía de la Estrella del Norte.

ROCA, J. L., 2001 - Economía y Sociedad en el Oriente Boliviano (Siglos XVI-XX), 678 pp.; Santa Cruz: Cotas Ltd.

VACA CHÁVEZ, F., 1913 - Informe elevado a la consideración del Supremo Gobierno por el Prefecto y comandante general, 104 pp.; Trinidad: sin pie de imprenta.

\section{Fuentes secundarias}

BARRAGÁN, R. \& ROCA, J. L., 2005 - Regiones y poder constituyente en Bolivia. Una historia de pactos y disputas. Cuaderno de Futuro 21, 458 pp.; La Paz: Informe del Desarrollo Humano, Programa de Naciones Unidas para el Desarrollo.

BECERRA CASANOVAS, R., 1992 - Símbolos departamentales, 17 pp.; La Paz: Offset Ltda.

BLOCK, D., 1997 - La cultura reduccional en los Llanos de Mojos, 260 pp.; Sucre: Historia Boliviana.

CÉSPEDES, L. R. de, 1942 - Simbolismo del Escudo del Beni. In: Homenaje al Beni en su primer centenario departamental (Escuela Profesional de Señoritas): 3; Buenos Aires: Salguero Editorial.

CORTÉS, J., 1989 - Notas al diario del P. Gumercindo Gómez de Arteche. In: JHS Misión de los PP Astraín, Mansanedo y Arteche c. a. 1888 (G. Gómez de Arteche, ed.): 34-44; Trinidad: Centro de Investigación y Documentación para el Desarrollo del Beni.

DÁvAlOS MENDOZA, F. J., 2006 - Escudo oficial del departamento del Beni. Historia oficial, 20 pp.; Trinidad: Prefectura del Departamento del Beni, Comisión de Límites.

FIFER, V., 1970 - The Empire Builders. A History of the Bolivia Rubber Boom and the Rise of the House of Suarez. Journal of Latin American Studies, 2 (2): 113-146.

GAMARRA, M. del P., 2007 - Amazonía Norte de Bolivia. Economía Gomera (1870-1940), 444 pp.; La Paz: Colegio Nacional de Historiadores de Bolivia, Producciones CIMA Editores.

GAMARRA, M. del P., 2010 - El desarrollo del concepto Beni: Proceso socio-histórico de formulación de la idea de Beni en la memoria colectiva de sus comunidades sociales. In: Cultura e identidad beniana (Justa Suárez, ed.): 15-37; Trinidad: Gobierno 
Departamental del Beni, Casa de la Cultura del Beni, Programa de Naciones Unidas para el Desarrollo, Agence Canadienne de Développement International, Programa de Investigación Estratégica de Bolivia.

GROFF GREEVER, J., 1987 - José Ballivián y el Oriente Boliviano, 224 pp.; La Paz: Empresa Editora Siglo Ltda.

GUITERAS, A., 2010 - Los indígenas benianos en el acceso a la propiedad de la tierra y la constitución de una sociedad de frontera en la Bolivia republicana, 1842-1915. Boletín Americanista, 60: 67-89.

GUITERAS, A., 2011a - A propósito de las leyes bolivianas de colonización de tierras baldías. La construcción de la sociedad beniana y el avance de la frontera interna (1880-1920). El Estado en América Latina. Recursos e imaginarios, siglos XIX-XXI (P. García Jordán, ed.): 59-94; Barcelona: Publicacions de Universitat de Barcelona.

GUITERAS, A., 2011b - Propiedad indígena y titulación de tierras en la Amazonía boliviana, 1842-1880. Caravelle. Cahiers du Monde Hispanique et luso-bresilien, 96: 35-50.

HOLLWEG, M. G., 1995 - Alemanes en el Oriente Boliviano, Tomo I, 482 pp.; Santa Cruz de la Sierra: Sirena.

LEHM, Z., 1999 - La búsqueda de la Loma Santa y la marcha indígena por el territorio y la dignidad, 151 pp.; Santa Cruz de la Sierra: Apoyo para el Campesino-Indígena del Oriente Boliviano, Centro de Investigación y Documentación para el Desarrollo del Beni, OXFAM América.

LIMPIAS SAUCEDO, M., 2005 [1942] - Los Gobernadores de Mojos, 282 pp.; Trinidad: Prefectura del Beni.

ROUX, J. C., 2000 - La Bolivie Orientale, 318 pp.; París: L'Harmattan. 